\title{
Shakespeares Hamlet im Englischuntericht der gymnasialen Oberstufe
}

\author{
Maria Eisenmann
}

\begin{abstract}
Zusammenfassung
Literarische Texte werden heute nicht mehr als selbstverständlich im Sprachunterricht akzeptiert. Gerade Shakespeare im Englischunterricht ist seit Jahrzehnten eines der umstrittensten didaktischen Themen. Die bei manchen bis heute fraglos akzeptierte „schönste Pflicht“ der ShakespeareLektüre auf der einen Seite und die wiederkehrende provozierende Frage „Warum gerade Shakespeare?" auf der anderen Seite markieren die extremen Positionen gegenüber dem Stellenwert Shakespeares in den Lehrplänen der Bundesländer. Wie die meisten Untersuchungen bestätigen, sind die verbreitetsten Shakespeare-Lektüren nach wie vor Macbeth und Julius Caesar. Erst mit großem Abstand folgen die anderen großen Tragödien wie z.B. Hamlet. ${ }^{1}$ Doch gerade Hamlet, das zu den meist gespielten Dramen auf deutschen Bühnen gehört, ist nicht einfach ein beliebiges klassisches Stück - es ist der Klassiker par excellence und sollte nicht nur deshalb einen viel bedeutenderen Stellenwert im Englischunterricht heute einnehmen.
\end{abstract}

Shakespeares Hamlet im Englischunterricht der gymnasialen Oberstufe

\section{Funktion und Ziel des Literaturunterrichts}

Immer wieder taucht in Fachkreisen die Frage auf, ob und warum Literatur in den Schulen behandelt werden soll. Wichtig ist die Literaturvermittlung vor allem, weil sie sich mit grundlegenden menschlichen Befindlichkeiten beschäftigt. Sie ist zwar gerade bei Shakespeare oft zeitverhaftet, scheint aber dennoch über die Jahrhunderte hinweg Identifikationsmöglichkeiten für Lernende zu bieten. Literatur liefert neben authentischem Material auch authentische Sprache aus ihrer Zeit, wobei zu berücksichtigen ist, dass der Rezipient mit der artifiziellen Sprache in Shakespeares Dramen einem Kunstprodukt begegnet. Texte, die eigentlich für den native speaker bestimmt sind, eröffnen dem Leser bzw. Lerner eine Vielfalt linguistischer Formen, Konventionen und Stilmittel. Durch intensives Lesen wird darüber hinaus auch der Wortschatz in der Fremdsprache erweitert. Anzumerken bleibt hierbei allerdings, dass Literatur nicht unbedingt

${ }^{1}$ Vgl. Ungerer 1982: 220. 
den Wortschatz vermitteln kann, den der Lerner wirklich braucht, weil dort häufig Vokabular verwendet wird, das sich nicht im Rahmen der Alltagssprache bewegt. Auf der anderen Seite kann gerade die poetische, literarische Sprache den Leser für Feinheiten und verschiedenartige Strukturen sensibilisieren.

Dies alles stellt Herausforderungen für die gegenwärtige Fremdsprachendidaktik dar. In der heutigen Unterrichtsgestaltung sind Kommunikationssituationen sehr stark gelenkt, wodurch der direkte und auch individuelle Zugang zur Literatur häufig ausbleibt. Unberücksichtigt bleibt dabei oft die Tatsache, dass sprachliche Kommunikation immer mehrdeutig ist und erst dadurch die Textanalyse und -interpretation ihren Reiz erfährt. Indem sprachliche Symbole immer wieder neu gedeutet und diskutiert werden können, erhält der Literaturunterricht seinen erkenntnistheoretischen und damit auch erzieherischen Wert. Hüllen kritisiert in diesem Zusammenhang die eingeengte Kommunikationssituation des schulischen Fremdsprachenunterrichts wie folgt:

\begin{abstract}
Viele Phänomene des kommunikativen Alltags kommen in der Schule gar nicht vor: Es gibt keine informatorischen oder thematischen Überraschungen; es gibt keine ernsthaften Mißverständnisse; es gibt keinen alltäglichen, sich selbst regulierenden Sprecherwechsel ... es gibt kein (oder nur wenig) wirkliches Betroffensein von dem, was gesagt wird oder was man selbst sagt. (Hüllen 1983: 169)
\end{abstract}

Um eine stärkere Orientierung am kommunikativen Alltag zu erreichen und wirkliche Gesprächsanlässe zu bieten, müsste die Besprechung literarischer Texte noch mehr Beachtung im Fremdsprachenunterricht finden. Gerade Literatur ist dazu geeignet, thematische Überraschung und wirkliches Betroffensein beim Lerner zu erzielen. Das ist in erster Linie darauf zurückzuführen, dass das Textverständnis immer auch an Erwartungen und Vorerfahrungen des Rezipienten geknüpft ist und dass jeder Leser durch seinen individuellen Hintergrund das Werk auf seine ihm eigene Weise wahrnimmt.

Ein Text steht immer in einem nicht wegzudiskutierenden Kontext. Der werkimmanenten Methode des New Criticism ist vorzuwerfen, dass sie eben diesen Kontext verleugnet und den literarischen Text in einen luftleeren Raum stellt. Der poststrukturalistische Dekonstruktivismus, der die Auseinandersetzung mit einem Text auf ein freies und autonomes Spiel der beschriebenen Bilder reduziert, hat in mancherlei Hinsicht zwar seine Berechtigung, es wäre jedoch schade, die aufschlussreichen und vielschichtigen Quellen kulturkundlicher Erkenntnisse unberücksichtigt zu lassen. Gerade diese machen nämlich die Mehrdimensionalität eines literarischen Textes im Gegensatz zum Sachtext aus. Somit treten Text und Kontext in ein komplexes Verhältnis zueinander:

Während die herkömmliche Fakten- und Realienlandeskunde eher einer organisierten Gruppenexkursion mit fixen Reiserouten und gedrängten Programmen gleicht, entspricht die literarisch betriebene Landesund Kulturkunde einer ungebundenen individuellen Entdeckungsreise mit reizvollen Abstechern, unerwarteten Begegnungen und subjektiven (auch affektiven) Erlebnissen. (Heller 1992: 29) 
Der Schüler soll also nicht Schritt für Schritt das Land auf kognitiver Ebene „erobern“, sondern offen und neugierig gemacht werden für kulturelle und gesellschaftliche Verknüpfungen. Dass Literatur dabei nicht zum Instrument der Landeskunde werden darf, darauf verweist Arno Heller:

In einer zunehmend technokratischen Zeit, die dazu neigt, alles zu instrumentalisieren, d.h. alles Erlernte nach seinen unmittelbaren Verwertungsund Anwendungsmöglichkeiten zu beurteilen, liegt der zentrale erzieherische Wert der Literatur, auch der fremdsprachlichen, zunächst zweifellos im Zurückdrängen der Zweckrationalität. (...) Auseinandersetzung mit der Literatur (ist) zu einem der letzten geistigen Freiräume geworden. (Heller 1992: 27)

Die angestrebten Ziele einer Verbindung von Literaturunterricht und kulturkundlichem Unterricht sollten daher eine Sensibilisierung und das Schaffen eines Problembewusstseins gegenüber der anderen Kultur und des anderen Zeithintergrundes sein. Literaturunterricht muss deshalb weit mehr sein als ein Medium für Sprachvermittlung bzw. landeskundliche Erkenntnisse. Zwar dient sie diesen beiden Disziplinen, aber nur unter der Voraussetzung, dass der Leser mit Interesse und Motivation an das Werk herantritt. Literatur als einer der letzten geistigen Freiräume soll und darf nicht instrumentalisiert werden und damit einer Zweckrationalität dienen. Lesen muss für den Schüler ein besonderes Erlebnis bleiben, wobei der Text in ein mehrdimensionales Wechselspiel aus Literatur-, Kultur- und Sprachvermittlung treten sollte.

\section{Besonderheiten des Dramenunterrichts}

Gerade der dramatische Text gewinnt laut Ahrens durch seinen außerordentlichen Wert für die kommunikative Kompetenz an enormer Bedeutung für den Englischunterricht heute und beeinflusst den Lehrer hinsichtlich der Auswahl der Lektüre:

The functional approach goes beyond the goal of simply understanding a literary text to that of improving linguistic competence and stimulating the communicative process. (Ahrens 1995: 425)

Die dramatische Wirklichkeit entsteht aus dem Zusammenwirken von Handlung, Sprache, Figuren, Zeit und Raum sowie der Bühnendimension. Der Text dient immer zugleich als Informationsträger und als Kommunikationsmedium.

Unter gattungsspezifischen Gesichtspunkten sind fiktionale Texte zunächst einmal als gleichrangig anzusehen. Dennoch ergeben sich für das Drama Eigenarten, die zu unterschiedlichen Interpretationsverfahren führen. Vom Fehlen der Erzählfunktion abgesehen, konfrontiert die dargestellte Figur den Rezipienten unmittelbar; die Situation konstituiert sich nur über Dialoge, da eine Erzählerinstanz fehlt, die vermittelnd und deutend in den Geschehensablauf eingreift. Der Dramenschreiber muss sich auf Haupttext (Dialoge) und 
Nebentext (Szenenanweisungen) beschränken. Außersprachliche Komponenten wie Mimik, Gestik, akustische und optische Effekte sowie Raum und Bühnenbild erlangen erhebliche Bedeutung. Durch den bewussten Gebrauch von gesprochener Sprache und die Verwendung von dramaturgischen Mitteln erweist sich ein Drama als ein literarischer Text ganz besonderer Art, gewinnt literaturdidaktisch eine außergewöhnliche Relevanz und erhält damit im Rahmen eines auf Text- und Medienkompetenz angelegten Unterrichts einen hohen Stellenwert. Reduziert man ein Drama auf sein literarisches Substrat, würde das eine Verkürzung bedeuten:

Das Drama ist im Gegensatz zur Epik und Lyrik nicht allein eine literarische Form, sondern literarische und gegenständliche Kunst zugleich.

(Glaap 1983: 15)

Neben kognitiven Zielen, d.h. bewusstem, kritischem Gebrauch der Zielsprache, bietet das Drama auch affektive Lernziele. Der Spielcharakter von Drama regt beispielsweise Kreativität an. Während früher der Zugang zu dramatischen Texten im Unterricht eher inhaltlich-thematisch ausgerichtet war, legte schon der Dramenunterricht der 80er und 90er Jahre immer mehr Wert auf eine schülerzentrierte Erarbeitung der Stücke. In vielen Schulen existieren seither Theatergruppen, die die Stücke bearbeiten, interpretieren, verändern und aufführen. So wird Dramenunterricht heute immer mehr in der Verschränkung von ästhetischer und pädagogischer Perspektive gesehen: „the connection between drama as an aesthetic and as an educational experience." (Bolton 1984: 140) Auch Bredella / Legutke unterstützen die schülerzentrierte Methode innerhalb des Literaturunterrichts (vgl. Bredella et al. 1985).

Kappe geht noch einen Schritt weiter und stellt die Methode vor, die Schüler direkt in das Dramengeschehen einzubeziehen und die Szenen direkt zu interpretieren. Er sieht einen hohen pädagogischen Wert darin, den Rezipienten von der Konsumentenrolle wegzubewegen und ihn zum permanenten Reflektieren darüber zu bringen, was sich im Drama abspielt (vgl. Kappe 1991). Eine wesentliche Aufgabe des Literaturunterrichts ist es, den Schülern den Zugang zu ungewohnten literarischen Erscheinungsformen zu eröffnen. Drama ist in dieser Hinsicht besonders wichtig, weil die Schüler in den Medien, besonders durch das Fernsehen, der Gattung Drama am meisten begegnen.

Es liegt auf der Hand, dass Dramen nicht geschrieben wurden, um gelesen, sondern um aufgeführt zu werden. Im Sinne von The play's the thing finden sie ihre gattungsentsprechende Behandlung überhaupt erst auf der Bühne, wodurch der dramatic approach an Bedeutung gewinnt. Durch die hermetische Beschäftigung mit dem geschriebenen Text stellt die Lektüre eines dramatischen Werkes für den Lerner eine ganz besondere Herausforderung dar, da er die Handlung, die sich im Drama eben nicht narrativ entfaltet, in seiner dramenspezifischen Dialogstruktur erfassen muss. Um sich dieser Herausforderung zu stellen, sollte in angemessener Weise auf den Aufführungscharakter eines Stückes eingegangen werden, wozu sich am 
besten ein Theaterbesuch eignet. Wenn dies aus organisatorischen Gründen nicht möglich ist, sollte zumindest die Aufzeichnung einer Aufführung auf Video bzw. DVD gezeigt werden.

\section{Die Verankerung Shakespeares in den Richtlinien der Fachlehrpläne}

In der Fachliteratur kann man hinsichtlich der Dramenbehandlung häufig vom "Sonderfall Shakespeare“ lesen und, obwohl Shakespeare der am meisten inszenierte Autor auf deutschen Bühnen ist, stellt sich immer wieder die Frage, ob und warum seine Werke im Unterricht gelesen werden sollten. Riggio beantwortet diese Frage mit dem Hinweis auf die Lehrpläne und betont die Aktualität des Autors:

[...] why focus on Shakespeare? [...] because they [Shakespeare's plays] are still one common factor in most contemporary educational curricula and because they have been restaged, adapted, changed, and revived at every period of English and American history. (Riggio 1999: 3)

Auch im Englischunterricht der gymnasialen Oberstufe hat die Lektüre von Shakespeares Dramen nach wie vor ihren festen Platz. Vor allem Julius Caesar, Macbeth, Romeo and Juliet und A Midsummer Night's Dream sind in den meisten Lehrplänen fest verankert. Viele Gegenstimmen machten vor allem auf die schwierige Sprache des Dichters aufmerksam. Aufgrund der historischen Distanz wurde zudem eine Identifikation der Lernenden mit den Shakespeareschen Figuren bezweifelt. Trotz dieser Kritik setzte sich Shakespeare als Unterrichtsgegenstand nicht nur im Englisch-, sondern auch im Deutschunterricht durch. Werk und Wirkung William Shakespeares bilden, auch historisch gesehen, ein besonderes Phänomen in den Schulen.

Shakespeare war schon immer ein fester Bestandteil des Englischunterrichts. Am Ende des 19. Jahrhunderts stand der Autor im Dienst der klassischen, humanistischen Bildung, da durch sein Werk, insbesondere durch sein dramatisches Werk, die Vermittlung universeller Lebensweisheit möglich war. Durch die Analyse moralischer Wertmaßstäbe sollte der Schüler zur Erkenntnis transzendenter Qualitäten geführt werden. Im Vordergrund standen die Historien, mit dem Ziel, den Schüler in die Geschichte menschlicher Tragik einzuführen. Die Lektüre wurde jedoch meist nur in Übersetzung gelesen.

Die Shakespeare-Rezeption in Deutschland weist ohnehin eine wechselvolle Geschichte auf, in der der Dichter für die unterschiedlichsten Interessen benutzt wurde. Sehr großen Einfluss hatte Shakespeare schon auf die Literaturtheorie der Aufklärung bei Gotthold Ephraim Lessing (vgl. 17. Literaturbrief 1759). Lessing zufolge orientiert sich das Drama Shakespeares an der Natur - ganz in Anlehnung an das antike Drama und im Gegensatz zum Drama der französischen Klassik. Ähnlich argumentieren die Dramatiker des Sturm und Drang, etwa Heinrich Wilhelm von Gerstenberg (Briefe über Merckwürdigkeiten 
der Litteratur, 1766/67), Johann Gottfried Herder (Von deutscher Art und Kunst, 1773) und Johann Wolfgang von Goethe (Rede zum Shäkespears Tag, 1771), in der er die Nähe von „Shakespeares Menschen“ zur „Natur“ beschwor. In dieser Zeit wurde der englische Dramatiker zum Prototyp des Genies und man sah ihn als dritten deutschen Klassiker neben Goethe und Schiller. Einen ähnlich großen Einfluss hatte Shakespeare nicht nur auf die deutsche Romantik, sondern auch auf die Dramentheorie des gesamten 19. Jahrhunderts. Shakespeare wurde „zu einem Vorbild auf der Suche nach einer nationalen kulturellen Identität, die sich von der französischen Dominanz emanzipiert.“ (Kullmann 2005: 12)

Die besondere Bedeutung Shakespeares für die Deutschen lässt sich nicht zuletzt an der bereits 1864 erfolgten Gründung der Deutschen ShakespeareGesellschaft ablesen. Unübersehbar ist aber auch die Flut der Eindeutschungen Shakespeares seit über 250 Jahren. ${ }^{2}$ Vor allem die Übersetzung von August Wilhelm Schlegel, Dorothea Tieck und Wolf Graf Baudissin (1796-1833), die bis ins 20. Jahrhundert hinein im Deutschunterricht fast ausschließlich benutzt wurde, ließ bei den deutschen Schülern keinen Zweifel aufkommen, dass es sich hier nicht um einen deutschen Dramatiker handelte. Auch zahlreiche deutsche Schriftsteller glaubten, eine Art Seelenverwandtschaft zwischen Shakespeares Texten und dem „deutschen Wesen“ zu erkennen, wie dies Friedrich Gundolf in seiner Schrift Shakespeare und der deutsche Geist (1911) darstellt. Der Grund für dieses nachhaltige Bild vom „deutschen Shakespeare“ (Ahrens 1982: 49) liegt jedoch nicht nur in der Schlegel-Tieck-Übersetzung begründet, sie liegt vor allem an der Identifikation der Deutschen mit Shakespeares Wesen, was Körbel im Jahre 1928 folgendermaßen beschreibt:

\begin{abstract}
Ja, durch eine (reichbelohnte!) willige Hingabe an seine Schöpfung, durch bindende Wahlverwandtschaft, durch die größere seelische Freiheit und Unvoreingenommenheit des deutschen Menschen ist er fast mehr 'unser', als er an der normalen seelischen Enge seiner konventionstreueren britischen Landsleute teil hat. (Körbel 1928: 106)
\end{abstract}

Nachdem Shakespeares Werk in der ersten Hälfte des 20. Jahrhunderts in erster Linie durch nationalsozialistische Erziehungsideale ideologisch missbraucht worden ist, blieb es auch in der zweiten Hälfte des 20. Jahrhunderts weiterhin ein nicht wegzudenkender Kernpunkt der deutschen Lehrpläne für Gymnasien sowohl in der BRD als auch in der DDR. Immer wichtiger wurde dabei die formalästhetische Interpretationsmethode und die Erfassung des Gesamtwerkes in der Originalsprache, wobei im Englischunterricht der DDR Shakespeare nicht im Original, aber auf Deutsch im Deutschunterricht gelesen wurde.

Mit Einführung der neuen Lehrpläne in fast allen Bundesländern ist die Behandlung der dramatischen Form sowohl im Grund- als auch im Leistungskurs vorgesehen. In der Regel schreibt der Leistungskurs - im Gegensatz zum Grundkurs - ein Drama Shakespeares vor. Die Untersuchungskriterien im

\footnotetext{
2 Bekannte Übertragungen der Dramen Shakespeares ins Deutsche sind die Ausgaben von Christoph Martin Wieland, Eduard Wilhelm Sievers sowie diejenige von Johann Heinrich Voß und seinen Söhnen Heinrich und Abraham Voß.
} 
Grundkurs, für den wenig verbindliche Vorgaben existieren, beschränken sich auf die Analyse der Handlung, der Charaktere, der Thematik, der Weltsicht und der Sprache. Im Leistungskurs werden sie erweitert auf Aufbau und Szenenfolge, Figurenkonstellation, Charakterisierungstechnik, Monolog, Dialog und andere dramatische Mittel sowie Bühne und Aufführungspraxis.

\section{Hamlet im Englischunterricht - zwischen Identifikation und kultureller Distanz}

Da die von Shakespeare dargestellten Grenzsituationen auf ganz besondere Weise fruchtbares und aktuelles Unterrichtsmaterial bieten, ist sein Werk auch heute noch eine pädagogische Herausforderung. Und dies gilt ganz besonders für Shakespeares Meisterwerk Hamlet, das nach Holger Klein als eines der komplexesten, rätselhaftesten, aber auch größten Dramen Shakespeares und als „Schlüsseltext der modernen abendländischen Kultur" gilt. (Klein; zit. in Poppe 2000: 142)

Es ist eine Rachetragödie, die sich mit dem Tod, mit der Frage nach der Schauspielkunst und auch mit der Frage nach politischer Verantwortung auseinandersetzt. Es gibt unzählig viele Interpretationsansätze, aber weder eine endgültige noch eine allgemeingültige. Das Stück bietet alles, was eine große Tragödie haben muss: großartige Monologe, einen psychologisierten, reflektierenden Helden mit tiefgründigen Gedanken, Liebesgeschichten, Familienkonflikte, die die innere Zerrissenheit zeigen, Geister, Mord und Rache, Wortgefechte, Duelle mit dem Schwert und die große alles vernichtende Todesszene am Ende des Dramas:

Hinterhalt und Intrigen bestimmen weitgehend die Handlungsweisen aufseiten der Hamlet-Gegner [...] auch Giftmischerei (Vorgeschichte) und eindrucksvolle Militärauftritte (Fortinbras) fehlen nicht in der zu jeder Zeit auch spannenden Vordergrundhandlung. (Poppe 2000: 145)

Doch das Stück ist viel mehr - es handelt vom inneren Konflikt des jungen Hamlet, der die Welt, in der er lebt, nicht mehr versteht und nicht weiß, wie er sich inmitten des Strudels von Korruption, Intrigen und politischen Machtspielen verhalten soll. Gerade die Vielschichtigkeit des Stückes liefert einen Anknüpfungspunkt, verschiedene didaktische Kriterien daraufhin zu überprüfen, inwieweit sich Shakespeares Charaktertragödie Hamlet als Lektüre in der gymnasialen Oberstufe eignet.

Häufig wurden Tragödien wie Hamlet, King Lear oder Coriolanus als zu lang erachtet, während Macbeth und Julius Caesar aufgrund des geringeren Umfangs schon seit den 60er Jahren als Lektüre gewählt wurden. (vgl. Schubel 1962: 155f.) In ihrer Studie Shakespeare im Leistungskurs Englisch weist Schmidt drauf hin, dass Hamlet mit Abstand nach Julius Caesar, Macbeth und The Merchant of Venice den vierten Platz in der kanonisierten Reihenfolge im Englischunterricht heute einnimmt. (vgl. Schmidt 2004: 57) Müller, der auf die große thematische 
Vielfalt des Stückes hinweist, erklärt die Scheu, Hamlet im Englischunterricht zu lesen wie folgt:

Der kanonische Charakter des Hamlet zeigt sich allerdings darin, dass das Drama vielfach auch im Deutschunterricht behandelt wird. [...] Gründe für die doch noch verbreitete Zurückhaltung gegenüber dem Drama mögen in dessen großer Länge und in den besonderen Schwierigkeiten liegen, die der Text dem Leser und Interpreten aufgibt. (Müller 2006: 21)

Doch selbst wenn Hamlet mit rund 4000 Zeilen Shakespeares längstes Drama ist, kann nicht allein der Umfang eines Dramas ausschlaggebend für die Wahl der Lektüre sein.

Die Frage nach der sprachlichen Anforderung ist sehr eng mit dem Umfangskriterium verknüpft. Landläufig gilt die Sprache in Julius Caesar als eher einfach, Hamlet als schwierig. Nach Hombitzer weisen die Tragödien eine „hohe Komplexität der Sprache“ auf, die Komödien gelten dagegen als „sprachlich weniger dicht“ (Hombitzer 1977: 77-92). Shakespeares Sprache ist grundsätzlich reich an poetischen Sprachmustern und rhetorischen Figuren und so ist aus Schülersicht wohl kein Shakespeare-Drama wirklich einfach $\mathrm{zu}$ verstehen. Daher macht es kaum einen Unterschied, ob eine Komödie, das altbewährte Drama Macbeth oder Hamlet als Schullektüre gewählt wird. Durch die Monologe bietet allen Schwierigkeiten zum Trotz gerade Hamlet die Möglichkeit für die Lernenden, die psychische Verfassung des Protagonisten zu erschließen und im gleichen Zuge zu erfahren, dass „ein essentieller Unterschied zwischen Hamlets sprachlichem Verhalten im Dialog und im Monolog (Selbstgespräch) besteht.“ (Müller 2006: 28) Man kann mit Schülern zusammen über die Bedeutung von Selbstgesprächen reflektieren und auf diesem Weg zu einem tieferen Verständnis von Hamlets Verhalten gelangen.

Sehr entscheidend für die Wahl der Lektüre ist das Kriterium der Handlungskomplexität. Die Handlung der Shakespeare-Dramen kann im Unterricht nicht in ästhetischer Hinsicht bewertet werden, sondern nur unter dem Aspekt der Zugänglichkeit für den Schüler. Im Gegensatz zu den komplexen Handlungen der Historien und den oft verwirrenden Handlungssträngen der Komödien erscheint Hamlet sehr überschaubar und gut verständlich. Dies liegt nicht zuletzt daran, dass nahezu alle Geschehnisse direkt mit der Haupthandlung verknüpft sind, so dass der Rezipient nicht den roten Faden verliert.

In den Komödien und Historien dagegen ist die Handlungsführung durchwegs mehrsträngig aufgebaut, wobei sub plot und main plot zum Teil nebeneinander herlaufen, sich aber dann unerwartet auf komplizierte Weise verschränken. [...] Erleichtert wird der Zugang zum Drama auch, wenn der Handlungsablauf durch eine Vielzahl äußerer Ereignisse markiert ist, die sich schon rein oberflächlich deutlich voneinander abheben, wie dies in Julius Caesar, Macbeth und Hamlet der Fall ist. (Ungerer 1982: 224)

Auch die Figurenvielfalt spielt für den jungen Leser eine entscheidende Rolle. In den Historien erscheinen das umfangreiche Personeninventar, die 
komplexen Familienbeziehungen sowie die vielen Titel und Amtsbezeichnungen eher hinderlich für einen guten Zugang zum Werk. Nicht nur durch die Personenvielfalt, sondern vor allem durch die vielen Verwechslungs- und auch Verkleidungsszenen könnte sich auch die Behandlung einer Komödie als eher schwierig gestalten. Im Drama Hamlet sind sowohl die Anzahl der Figuren als auch die familiären Verbindungen sehr überschaubar und transparent.

Die Shakespeare-Forschung ist uneins darüber, ob Shakespeares Werk als zeitlos bezeichnet werden kann oder ob es doch sehr in der elisabethanischen Epoche verhaftet ist. Wie dem auch sei, es ist mit Sicherheit sehr förderlich für den Unterrichtsverlauf, wenn der Lerner Informationen über die Bedingungen des Theaterbetriebes um 1600, politisch-soziale Hintergründe und gesellschaftliche Konventionen der elisabethanischen Zeit erhält. Hier stellt sich die Frage, ob ein über 400 Jahre alter Text für die heutigen Schüler überhaupt noch zugänglich ist? Oder anders gefragt, ist die kulturelle Distanz nicht bei jedem Shakespeare-Drama - ob Historie oder Tragödie - schon vorprogrammiert?

Bei den Historien entsteht die kulturelle Distanz in zweierlei Hinsicht zum einen geht es um einen historischen Stoff aus der Zeit des englischen Mittelalters, zum anderen um die dramatische Bearbeitung dieses Stoffes, der zudem die Geschichtsauffassung der Tudorzeit reflektiert (vgl. Habicht 1977: 46f.). Um all die Fragen zum historischen Hintergrund klären zu können, wäre eine Fülle an Information notwendig, was den zeitlichen Rahmen einer Lektürebesprechung im Unterricht in jedem Fall sprengen würde. Obwohl auch die meisten Tragödien historische Stoffe behandeln, sind sie für den Schüler eher fassbar als die Historien, denn „im Vergleich zu den Historien wird bei den Tragödien die kulturelle Distanz gleichsam 'überspielt' " (Ungerer 1982: 227). Das liegt nicht zuletzt daran, dass die privat-menschliche Ebene in den Tragödien in den Vordergrund gerückt und der Stoff somit aus seiner historischen Bindung herausgelöst wird (vgl. Heck et. al. 1978: 376). Die Thematik der Tragödien ist meist allgemeingültig und kaum zeitbezogen; sie handeln von menschlichen Grundbefindlichkeiten und Grenzsituationen, was dem Schüler eine Identifikation erleichtert.

Dass gerade Shakespeares Hamlet identifikatorisches Potential liefert, darauf verweist schon Sigmund Freud in seinem Hamlet-Aufsatz:

Der Zuschauer ... will fühlen, wirken, alles so gestalten wie er möchte, kurz Held sein, und die Dichter-Schauspieler ermöglichen ihm das, indem sie ihm die Identifizierung mit einem Helden gestatten. Sie ersparen ihm auch etwas dabei, denn der Zuschauer weiß wohl, daß solches Betätigen seiner Person im Heldentum nicht ohne Schmerzen, Leiden und schwere Befürchtungen, die fast den Genuß aufheben, möglich ist; er weiß auch, daß er nur ein Leben hat und vielleicht in einem solchen Kampf gegen die Widerstände erliegen wird. ... Das erste dieser modernen Dramen ist der Hamlet. (Freud 1969: 163-166)

Im aristotelischen Sinne von prodesse et delectare ist die Voraussetzung für den Genuss die Illusion, die auf der Bühne gespielt wird und das Leiden des Rezipienten wird nicht nur dadurch erträglich, dass es ein anderer ist, der auf 
der Bühne leidet, sondern auch durch die Tatsache, dass sich der Zuschauer des Spielcharakters bewusst ist.

Dabei eröffnen affektive Zugänge zum dramatischen Text häufig erst die Voraussetzung zu einem vertieften Verständnis. Nach Bergmann / Kroth sind Empathie, Toleranz, Perspektivenübernahme und Interaktionsfähigkeit einige der Schlüsselkompetenzen, die durch ganzheitliche, kreative Methoden erworben werden können (vgl. Bergmann / Kroth 2002: 7). Die Fähigkeit, verschiedene Perspektiven zu erkennen ist vor allem dann wichtig, wenn es darum geht, unterschiedliche Deutungsmöglichkeiten eines Textes bzw. verschiedene Interpretationen nebeneinander zu betrachten. Ein dramatischer Text, der immer wieder Hintergrund für Spielanlässe bietet und somit die Darstellung von Beziehungen einzelner Charaktere zueinander bzw. den Ausdruck von Stimmungen wiederzugeben vermag, kann laut Spinner zu „einem Erprobungsfeld für Perspektivenübernahme und das Ausloten der eigenen Identität" werden. Dies liegt nicht zuletzt daran, dass man damit experimentiert, „ein anderer zu sein. Aber man spielt immer sich selbst. Die Grenzen zwischen Ich und Anderem werden durchlässig." (Spinner 2001: 5)

Besonders bei jungen Lesern kann man altersbedingt ein ausgeprägtes Bedürfnis nach eben dieser Art von Identifikation und nach engagierter Sympathie feststellen. (vgl. Nickel 1975: 339f.) Dies kann im Dramenunterricht genutzt und sollte bei der Lektüreauswahl berücksichtigt werden. Friedrich Ungerer zufolge herrscht ,ein relativ großes Maß von Engagement für Brutus, für Hamlet (und Ophelia), eventuell auch für Romeo und Juliet. [...] Geringeres Engagement gilt [...] den Komödien, da hier selbst relativ sympathische Figuren durch die Artifizialität der dramatischen Darbietung in die Distanz rücken." (Ungerer 1982: 231)

Müller betont, dass Hamlet insbesondere aufgrund seiner Thematik sehr gut geeignet ist:

Auch von der Thematik her ist das Stück geeignet, Rezipienten im Alter der Adoleszenz anzusprechen, hat es doch einen jungen Protagonisten, der sich im Widerstreit mit der Generation der Eltern und der Gesellschaft befindet und in eine Krise gerät, die alle Beziehungen, speziell die zur Mutter und zur Geliebten, erschüttert und ihm tiefgreifende Identitätsprobleme bereitet. (Müller 2006: 21)

Gerade die schillernde Komplexität von Hamlets Charakter, die Problematik des Erwachsenwerdens und auch das ambivalente Verhältnis von Tragischem und Komischem erhöhen die unendliche Vitalität und Lebensechtheit der Bühnengestalt, mit der sich die Zuschauer über vier Jahrhunderte hinweg identifiziert haben.

\section{Umsetzungsmöglichkeiten der Lernziele}

Die große Bedeutung von Shakespeares Hamlet in der gymnasialen Oberstufe heute ist nicht zuletzt auch auf unzählige Theateraufführungen, Verfilmungen, 
moderne Adaptionen und Transformationen in verschiedene andere Medien und Genres wie Film, Hörspiel oder Erzählung zurückzuführen. (vgl. Walch 2004) Im Folgenden sollen nun einige didaktisch-methodische Möglichkeiten dargestellt werden, die zur Umsetzung der oben genannten Lernziele beitragen.

\subsection{Das Klassenzimmer als Bühne}

Die performanz-orientierte Herangehensweise an die Werke Shakespeares geht vornehmlich auf Gibson (1998; 2000) und Stredder (2004; 2006) zurück. Sie propagieren die Methode des workshop oder dramatic approach, die sie als Potenzial einer ganzheitlichen Annäherung an den dramatischen Text verstehen. Dabei geht es nicht darum, den gesamten Text im Klassenzimmer zu einer Aufführung zu bringen, sondern um eine intensive Auseinandersetzung mit einzelnen Stellen des Dramas. Das Ziel ist dabei, alle Schüler einzubeziehen und sie am Interpretationsfindungsprozess zu beteiligen. Das ganzheitliche Verständnis wird durch einen experimentellen Zugang zu Gestik, Mimik und Sprache erreicht.

Im Drama Hamlet bieten sich hier vor allem die Monologe der Hauptfigur an. Die Lerner sollten Textzeile für Textzeile artikulieren, sich dabei im Klassenraum bewegen und zum Gesprochenen gestikulieren. Dabei sollte es den Schülern freigestellt sein, ob sie den jeweiligen Monolog alleine oder dialogisch mit einem Partner umsetzen wollen. Um die Wirkung ihrer Stimmen auszuprobieren, sollte die Lehrkraft die Schüler dazu anhalten, mit Geschwindigkeit, Lautstärke und Betonung zu experimentieren. Auch die räumlichen Gegebenheiten des Klassenzimmers sollten in den experimentellen Zugang integriert werden.

\subsection{Hamlet im Film}

Die Geschichte der Shakespeare-Adaptionen durch den Film ist fast genauso alt wie das Medium Film selbst. Es gibt mehr als 20 Kinofilme, die unter dem Titel Hamlet erschienen sind sowie eine Reihe von Fernsehfilmen. Daneben gibt es gut 50 Filme, die das Wort Hamlet im Titel enthalten. Das Drama Hamlet avancierte nicht zuletzt aufgrund seiner sehr unterschiedlichen Verfilmungen zu einer der bekanntesten Tragödien Shakespeares, und da die Erziehung zu Medienkompetenz, wozu auch Filmanalyse zu rechnen ist, in nahezu allen Lehrplänen der Bundesländer gefordert wird, drängt sich der Einsatz von Verfilmungen im Englischunterricht geradezu auf.

Doch im Gegensatz zum traditionellen Literaturunterricht, der gewöhnlich nur eine Filmversion zum Vergleich mit der literarischen Vorlage heranzieht, ist es sinnvoller mehrere Filmvarianten miteinander zu vergleichen, da diese Art der Kontrastierung mehrerer Interpretationsansätze ein deutlich größeres Verständnis des Originaltextes gewährleistet. Auf diesen Zusammenhang verweist auch schon Hombitzer 1986:

Verschiedenartige Interpretationen zwingen dazu, sowohl den Text kritisch zu befragen, als auch den jeweiligen Zeithintergrund mit seinen 
Interessenschwerpunkten zu berücksichtigen. Dabei wird die Frage nach den eigenen erkenntnisleitenden Interessen relevant. (Hombitzer 1986: 1010)

Um den Blick der Lernenden sowohl für den Shakespeareschen Text als auch für die unterschiedlichen Interpretationsansätze auszubilden und zu entfalten, bietet sich aus didaktischer Sicht die vergleichende Analyse verschiedener Filmversionen mit der Fokussierung thematischer Schwerpunkte an.

Nach Volkmann leben unsere Schüler heute immer noch „mit dem vom tief verwurzelten Geniekult genährten Trugschluss, es gäbe eine philologisch exakt rekonstruierbare Urfassung des Hamlet, 'wie Shakespeare sie gewollt hat"“ (Volkmann 1997: 32). Die Gefahr, nur eine Filmvariante auszuwählen und diese parallel zur Lektüre des Dramas zu zeigen, wäre eine Verengung der Sichtweise, da so der Fokus auf nur eine Form der Interpretation begrenzt wird und der Lernende fälschlicherweise von „einer richtigen Interpretation“ ausgeht.

Von den vielen Hamlet-Adaptionen eignen sich die Versionen von Laurence Olivier (1948), Franco Zeffirelli (1990), Kenneth Branagh (1996) und Michael Almereyda (2000) ganz besonders zum Vergleich. Da der vollständige Einsatz eines solchen Filmes zumeist an der Länge scheitert, muss man sich in der Kontrastierung auf zentrale Szenen beschränken. Hier bieten sich der „to be or not to be"-Monolog (III, i), die mousetrap-Szene (III, ii) oder Hamlets Zögern (III, iii) an, da gerade hier die Doppeldeutigkeit der Situation und die innere Zerrissenheit der Figur Hamlets zum Tragen kommen. ${ }^{3}$

\subsection{Hamlet im Internet}

Gibt man über eine Suchmaschine im Internet den Begriff „Hamlet“ein, so erhält man eine unüberschaubare Masse an Einträgen. Sehr gute Nutzungsempfehlungen für die gezielte Suche nach Portalen, Lehrmaterialien, Originaltexten, Festivals, Theatern, Multimedia-Ansätzen, Foren, Freiarbeitsmaterialien und Hintergrundinformationen finden sich bei Baier (2006). Um den Schülern einen allgemeinen Eindruck von der Theaterlandschaft zur Zeit Shakespeares zu geben, empfiehlt sich ein Besuch der homepage des Globe Theatre in London. Eine Möglichkeit, sich mit Hamlet im Internet auseinanderzusetzen, ist ein Web Quest, bei dem die Schüler mit den Anweisungen, die sie per Internet erhalten, sehr autonom arbeiten können. Ein Web Quest gliedert sich in der Regel in sechs Schritte: Introduction - Task - Internet Sources - Process - Guidance - Conclusion. Einen guten Weg zur selbstständigen Arbeit bietet zudem die Erstellung eines Wikis ${ }^{4}$, an dem die gesamte Klasse mitarbeiten kann. Durch

\footnotetext{
${ }^{3}$ Neben diesen Film-"Klassikern" eignen sich für den modernen Englischunterricht auch weitere Filmvarianten. Man könnte z.B. den Musikclip Where the Wild Roses Grow von Nick Cave und Kylie Minogue vorspielen und die Schüler Parallelen zu Shakespeares Hamlet herausarbeiten lassen. Denkbar wäre auch der Vergleich mit dem Sketch aus Monty Python's Flying Circus, Episode 43 von 1974, der direkt Bezug zu Hamlets "to be or not to be"-Monolog nimmt.

${ }^{4}$ Der Begriff „Wiki“ leitet sich von „Wiki wiki“ ab, was auf Hawaiisch „schnell“ heißt. „Wiki“ ist die Kurzform für WikiWikiWeb.
} 
das Erstellen einer gemeinsamen Website zu Shakespeares Hamlet entsteht dann eine große Menge einfach zugänglichen und durchsuchbaren Wissens. Der besondere Wert eines Wikis besteht darin, dass seine Benutzer nicht nur die Seiten navigieren und ansehen, sondern gleichzeitig auch bearbeiten können.

Im Internet gibt es mittlerweile auch viele Parodien auf die Texte Shakespeares, allen voran auf den berühmten „to be or not to be“-Monolog, den Schüler einer 12. Klasse aus der Sicht verschiedener Personen geschrieben haben, wie z.B. The Stock Broker's Soliloquy: ${ }^{5}$

To buy or not to buy, that is the decision;

Whether 'tis nobler in the mind to suffer

The ups and downs of the unpredictable stock market,

Or to take arms against a sea of merciless profiteers

And by getting the inside news, become a millionaire.

To relax; to sleep, no way,

And by no sleep we mean to swallow up all

The caffeine pills, and follow the trend of the market

That will go up or down a thousand points

By the end of the day.

'Tis due to our ambition

That we find ourselves in such headaches.

To relax, to sleep;

To sleep; perchance to lower blood pressure;

Ay, there's the bell,

For in this dreadful and golden time comes to a finish, When we have complete the handling of our stocks, Must give us a final balance; there's the result

That makes us go to heaven or to hell.

Mit diesen web spoofs kann sich der Lernende kreativ-spielerisch an Originalzitate aus Hamlet annähern. Die Aufgabe für die Schüler könnte dann darin bestehen, die Parodien zu lesen und die beste auszuwählen oder eine eigene (alleine, in Partner- oder Gruppenarbeit) zu schreiben. Unter The funnies finden sich auf der gleichen Seite weitere originelle Ideen, um einen Zugang zu Shakespeares Hamlet herzustellen, z.B. ein Hagar Cartoon oder The Soliloquy Rap from 'Hamlet':

\subsection{Hamlet interdisziplinär}

\section{Hallet zu Folge}

scheint ein einzelnes Unterrichtsfach, und sei es das Leistungsfach Englisch, dem kulturellen Phänomen Shakespeare kaum gerecht werden zu können. Shakespeare stellt deshalb keine geringere Frage als die nach einer transnationalen und transkulturellen Rekonzeptualisierung des Literaturunterrichts, in dem es weder einen englischen noch einen deutschen Shakespeare gibt, sondern einen europäischen." (Hallet 2006: 222)

\footnotetext{
${ }^{5}$ Vgl. www.angelfire.com/oh/Pretzel/.
} 


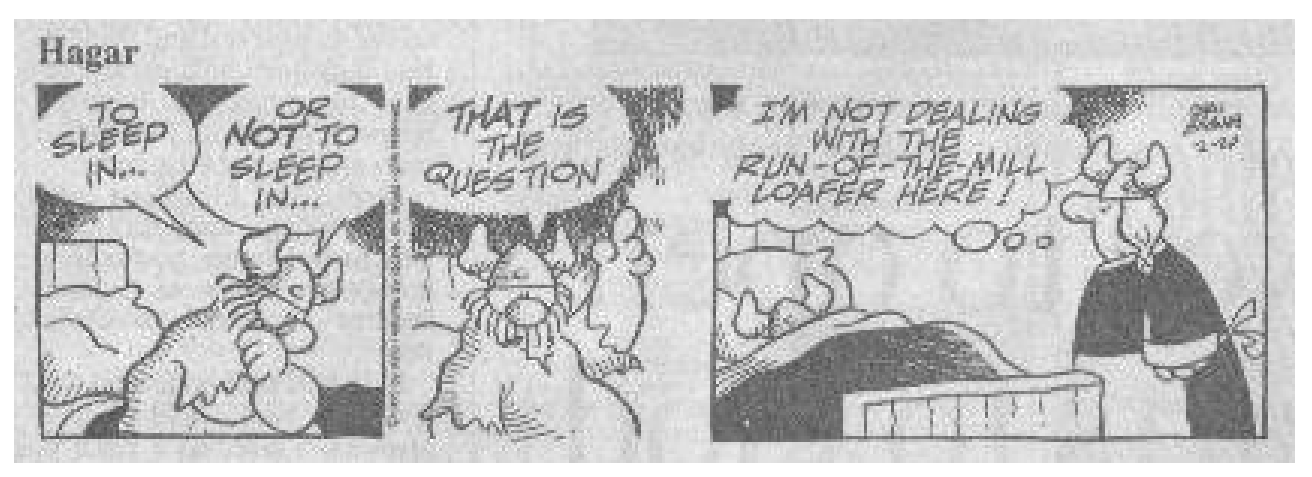

Abbildung 1: Hagar Cartoon

Was Shakespeares Hamlet betrifft, bietet sich vor allem der Deutsch- und Kunstunterricht für fächerübergreifendes bzw. fächerverbindendes Arbeiten an.

Die Lehrpläne für den Deutschunterricht in allen Bundesländern schlagen vor allem für die gymnasiale Oberstufe - die Behandlung von Shakespeare vor, wobei keine Verpflichtung besteht, sich mit dem Dichter auseinanderzusetzen. Dabei spielt die Shakespeare-Rezeption im Sturm und Drang und in der Klassik eine Hauptrolle. Die Lektüre einer Ganzschrift erfolgt oft nur im Leistungskurs, wobei hier vor allem Hamlet auf Deutsch gelesen wird. Für interdisziplinäres und intertextuelles Arbeiten bietet sich im Deutschunterricht insbesondere die Lektüre Heiner Müllers Adaption Hamletmaschine an, durch das die Schüler zu einer Auseinandersetzung mit der Teilung und Wiedervereinigung Deutschlands geführt werden.

Im Kunstunterricht können Teilaspekte zu Shakespeares Hamlet an verschiedenen Epochen der Kunstgeschichte vermittelt werden. Die meisten Bezüge zum Drama finden sich in Werken des 18. und 19. Jahrhunderts, wobei die englischen Präraffaeliten des viktorianischen Jahrhunderts eine ausgeprägte Vorliebe zum Ophelia-Motiv zeigten. Dem Tod Ophelias setzt John Everett Millais 1852 ein beispielloses Denkmal, in dem er mit seiner Darstellung das Bild der „schönen Toten“ im Wasser liegend zeigt. Ein sehr ähnliches Bild in Form einer Graphik schuf Eugène Delacroix in Frankreich 1853. Im Hinblick auf eine fächerverbindende Projektarbeit zu Shakespeares Hamlet können die Lernenden durch Bildanalysen die verschiedenen Sichtweisen und Interpretationen bis ins 20. Jahrhundert hinein betrachten. Dazu empfiehlt es sich, weitere Kunstwerke hinzuzuziehen, um anschließend über möglichst viele Parallelen und Differenzen diskutieren zu können. Gerade das Motiv der toten Ophelia lässt sich auch noch in der zeitgenössischen Kunst finden, z.B. als Fotografie von Gregory Crewdson Untitled (Ophelia) von 1998/2002 oder von Izima Kaoru Tomita Yasuko, Megurogawa de, Prada no wanpisu o kite von 1997.

Für einen direkten Vergleich des Originaltextes, der Kunst im 19. Jahrhundert und heutigen Darstellungsweisen eignen sich auch Musikvideoclips, z.B. Nick 


\section{The SOLILOOUY RAP from "HAMLET"}

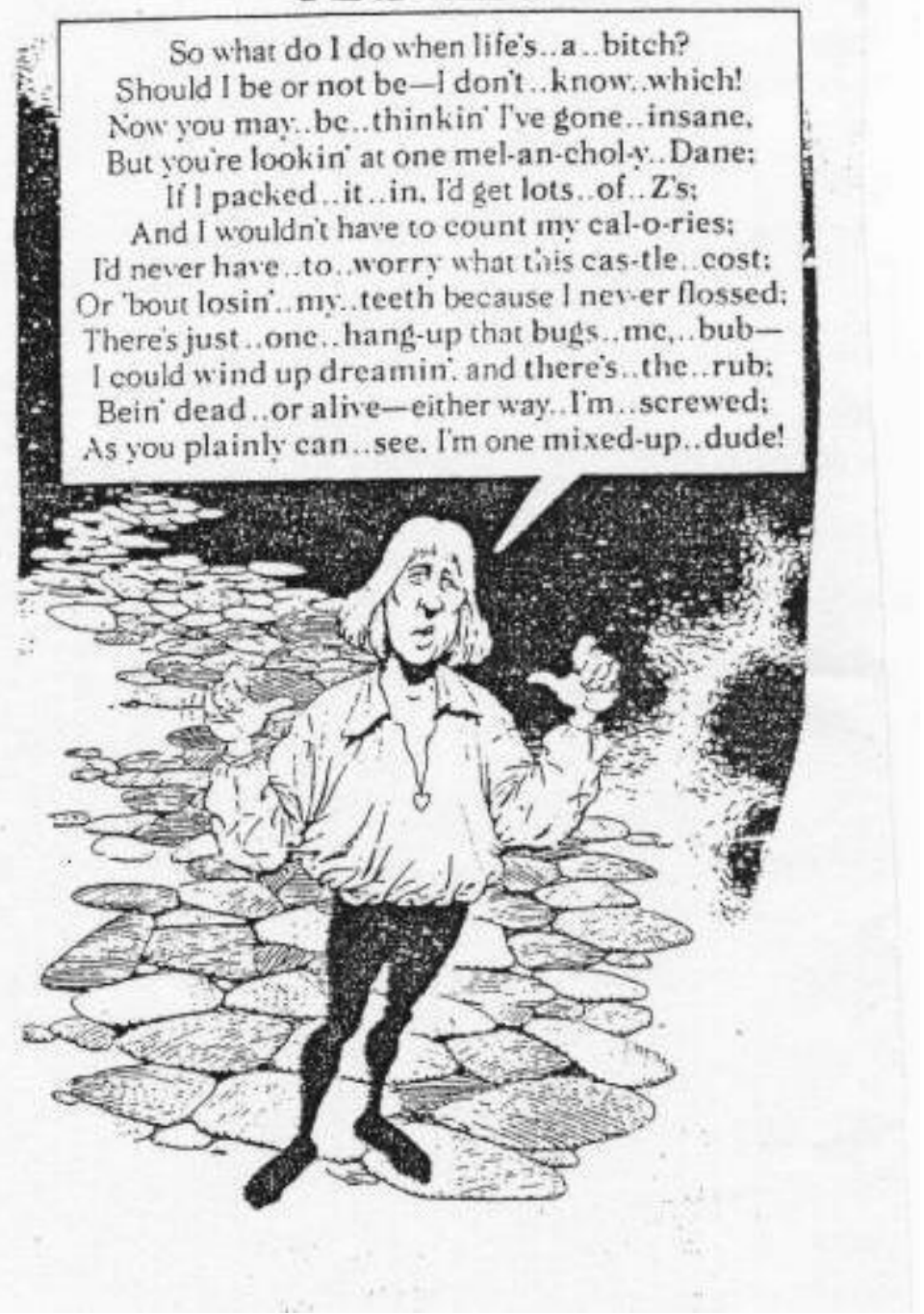

Abbildung 2: Soliloquy Rap from 'Hamlet' 
Caves und Kylie Minogues Where the Wild Roses Grow von 1995 oder Britney Spears Everytime von 2004, die beide das Motiv der schönen, nackten im Wasser liegenden Frau aufnehmen. (vgl. Wenrich 2006: 239ff.)

\section{Resümee}

Es liegt auf der Hand, dass eine angemessene Textauswahl die Grundlage für einen erfolgreichen Literaturunterricht ist. Daher muss sich die Auswahl einer Lektüre immer an der Lerngruppe orientieren, d.h. an ihren Bedürfnissen, Fähigkeiten, Interessen, an dem kulturellen Hintergrund und dem Sprachniveau. Wichtig ist dabei die Frage, ob mit dem Text Interesse geweckt werden kann. Durch die Vitalität und Komplexität der Figur Hamlets werden sowohl die Neugier als auch eine hohe Lesemotivation beim Lernenden gefördert. Der Schüler erhält ein Angebot von wertvollen Modellfällen des Lebens und der Wirklichkeit. Gerade im schulischen Kontext erscheint es demzufolge wichtig, die durchaus jugendnahen und zeitlosen Themen des Stückes aufzuzeigen und auf die Komplexität der Titelfigur zu verweisen.

Die Behandlung Shakespeares Hamlet im Unterricht ermöglicht neben der Erweiterung linguistischer Fähigkeiten und der Veranschaulichung der dramatischen Form lebhafte Diskussionen und den Zugang zum dramatic approach. Sie gewährleistet neben einem fruchtbaren Einsatz von Einzel, Gruppen- und Partnerarbeit auch interdisziplinäres sowie intertextuelles Arbeiten und verspricht dadurch einen beträchtlichen Gewinn an Text- und Medienkompetenz.

\section{Bibliographie}

Ahrens, Rüdiger (ed.) (1977): Anglistik \& Englischunterricht. Shakespeare im Unterricht. Trier: Volksfreund-Druckerei

Ahrens, Rüdiger (ed.) (1982): Shakespeare. Didaktisches Handbuch. Bd. 1-3. München: Fink-Verlag

Ahrens, Rüdiger (1982): Shakespeare in der Literaturdidaktik. In: Ahrens, Rüdiger (ed.): Shakespeare. Didaktisches Handbuch. Bd. 1. München: Fink-Verlag, 43-68

Ahrens, Rüdiger (1995): Curricular Goals of Reading Modern Drama: Textual Strategies of Samuel Beckett and Harold Pinter. In: Riehle, Wolfgang; Keiper, Hugo (eds.): Anglistentag: Proceedings of the Conference of the German Association of University Teachers of English. Bd. XVI. Tübingen: Niemeyer Verlag, 421-438

Baier, Jochen (2006): Shakespeare und das Internet. In: Petersohn, Roland; Volkmann, Laurenz (eds.): Shakespeare didaktisch I. Neue Perspektiven für den Unterricht. Tübingen: Stauffenburg, 135-146 
Bergmann, Birgit / Kroth, Olivia (2002): Much is the Force of Heaven-bred Poesy. In: Der Fremdsprachliche Unterricht Englisch. 65, 2002, 7

Bolton, Gavin M. (1984): Drama as Education. An Argument for Placing Drama at the Centre of the Curriculum. Essex: Longman

Bredella, Lothar / Legutke, Michael (eds.) (1985): Schüleraktivierende Methoden im Fremdsprachenunterricht Englisch. Ferdinand Kamp: Bochum

Brusch, Wilfried (1986): Text und Gespräch in der fremdsprachlichen Erziehung. Hamburg: ELT

Edens, Walter (1977): Teaching Shakespeare. Princeton, New Jersey:

Princeton University Press

Freud, Sigmund (1969): Bildende Kunst und Literatur. Bd. X. Frankfurt/ Main: Fischer-Studienausgabe, 161-168

Gibson, Rex (1998): Teaching Shakespeare. Cambridge: Cambridge University Press

Gibson, Rex (2000): Stepping into Shakespeare. Cambridge: Cambridge University Press

Gibson, Rex (2000): Active Methods in Shakespeare Classrooms. In: Der Fremdsprachliche Unterricht Englisch 46, 17-21

Glaap, Albert-Reiner (1983): Kurzdramen und kurze Dramen zeitgenössischer Autoren. Vorschläge für Grund- und Leistungskurse im Englischunterricht. In: Die Neueren Sprachen 82, 151-169

Glaap, Albert-Reiner (1995): Introduction. In: Riehle, Wolfgang / Keiper, Hugo (eds.): Anglistentag: Proceedings of the Conference of the German Association of University Teachers of English. Bd. XVI. Tübingen: Niemeyer Verlag, 417-419

Habicht, Werner (1977): Zum Shakespeare-Bild heute - Tendenzen der neueren Shakespeare-Forschung. In: Ahrens, Rüdiger (ed.): Anglistik und Englischunterricht. Shakespeare im Unterricht. Trier: Volksfreund-Druckerei, 39-52

Hallet, Wolfgang (2006): Shakespeare im Deutschunterricht. In: Petersohn, Roland / Volkmann, Laurenz (eds.): Shakespeare didaktisch I. Neue Perspektiven für den Unterricht. Tübingen: Stauffenburg, 207-224

Heck, Hans / Müller, Monika (1978): Die Historien. In: Schabert, Ina (ed.): Shakespeare-Handbuch: Die Zeit - Der Mensch - Das Werk - Die Nachwelt. Stuttgart: Alfred Kröner-Verlag, 365-382

Heller, Arno (1992): Zur Problematik interkultureller Literaturvermittlung im Englischunterricht der Sekundarstufe II. In: Der fremdsprachliche Unterricht 1-2, 26-30

Höfele, Andreas (2000): Hamlet. In: Lengeler, Rainer (ed.): Interpretationen Shakespeares Dramen. Stuttgart: Reclam, 238-272

Hombitzer, Eleonore (1986): Die Shakespeare-Verfilmung im Englischunterricht der gymnasialen Oberstufe. In: Ahrens, Rüdiger (ed.): 
William Shakespeare, Didaktisches Handbuch. Bd. 3, München: Fink, 987-1013

Hombitzer, Eleonore (1977): Shakespeare-Lektüre in der reformierten gymnasialen Oberstufe - unter besonderer Berücksichtigung von Grundkursen. In: Ahrens, Rüdiger (ed.): Anglistik und Englischunterricht. Shakespeare im Unterricht. Trier: Volksfreund-Druckerei, 77-91

Hüllen, Werner (1983): Über das allmähliche Verfertigen von Sprachregeln. In: Der fremdsprachliche Unterricht, 165-172

Kappe, Gerhard (1991): Neue Möglichkeiten der Drameninterpretation. Zeitgenössische englische Dramen als Aufführungsmedien in schüleraktivierenden Arbeitsweisen. Würzburg: Königshausen \& Neumann

Kliman, Bernice W. (2001) (ed.): Approaches to Teaching Shakespeare's

Hamlet. New York: The Modern Language Association of America

Körbel, Hans (1928); Zur Shakespeare-Lektüre an den Höheren Schulen. In:

Die Neueren Sprachen 36, 106-109

Küpper, Reiner (1982): Shakespeare im Unterricht. Geschichte, Konzeptionen, Tendenzen. Würzburg: Königshausen und Neumann

Kullmann, Thomas (2005): William Shakespeare. Eine Einführung. Berlin: Erich Schmidt

Nickel, Horst (1975): Entwicklungspsychologie des Kindes- und Jugendalters. Bd 2. Bern: Huber

Müller, Wolfgang G. (2006): Sieben Zugänge zu Shakespeares Hamlet. In: Petersohn,

Roland / Volkmann, Laurenz (ed.): Shakespeare didaktisch II. Ausgewählte Dramen und Sonette für den Unterricht. Tübingen: Stauffenburg, 21-35

Poppe, Reiner (2000): William Shakespeare. Stuttgart: Reclam

Riehle, Wolfgang / Keiper, Hugo (eds.) (1995): Anglistentag: Proceedings of the Conference of the German Association of University Teachers of English. Bd. XVI. Tübingen: Niemeyer Verlag

Riggio, Milla Cozart (ed.) (1999): Teaching Shakespeare through Performance. New York: The Modern Language Association of America

Schabert, Ina (ed.) (1978): Shakespeare-Handbuch: Die Zeit - Der Mensch Das Werk - Die Nachwelt. Stuttgart: Alfred Kröner-Verlag

Schmidt, Isolde (2004): Shakespeare im Leistungskurs Englisch. Eine empirische Untersuchung. Frankfurt/M: Lang

Schubel, Friedrich (1962): Shakespeare-Lektüre im Unterricht. In: Mitteilungsblatt des ADNV 15, 153-167

Spinner, Kaspar (2001): Spielszenen im Deutschunterricht. In: Praxis Deutsch 166, 4-9

Stredder, James (2004): The North Face of Shakespeare: Activities for Teaching the Plays. Stratford-Upon-Avon: Wincot Press 
Stredder, James (2006): Shakespeare spielen - das Klassenzimmer als Bühne. In: Petersohn, Roland / Volkmann, Laurenz (ed): Shakespeare didaktisch I. Neue Perspektiven für den Unterricht. Tübingen: Stauffenburg, 21-42

Ungerer, Friedrich (1982): Der Shakespeare-Kanon für den Schulunterricht. In: Ahrens, Rüdiger (ed.): Shakespeare. Didaktisches Handbuch. Bd. 1, München: Fink-Verlag, 219-239

Volkmann, Laurenz (1997): The ten Hamlets: eine performance-orientierte Methodik der Schüleraktivierung. In: Der fremdsprachliche Unterricht Englisch 6, 32-35

Walch, Günter (2004): Hamlet. Bochum: Kamp

Wenrich, Rainer (2006): Shakespeare (nicht nur) im Kunstunterricht. In: Peterson, Roland; Volkmann, Laurenz (eds.): Shakespeare didaktisch I. Neue Perspektiven für den Unterricht. Tübingen: Stauffenburg, 239-256 